\section{LONG-TERM RESULTS OF CARDIAC TRANSPLANTATION IN PATIENTS OLDER THAN SIXTY YEARS}

Advanced age has traditionally been a contraindication to cardiac transplantation. We have, however, offered cardiac transplantation to patients older than 60 years with end-stage heart failure if they were otherwise acceptable candidates. From 1985 to 1994, 527 patients underwent cardiac transplantation. Among these patients, 101 were older than 60 years at transplantation. The mean follow-up of this group is 6 years. Patients older than 60 years had significantly fewer rejection episodes per patient than those who were younger than 60 years at transplantation $(1.9 \pm 1.3$ vs $2.6 \pm$ $1.8, p=0.009$ ). No difference in the number of infectious complications per patient was detected between the two groups. Both short-term and longterm survival after transplantation were significantly lower for patients who were older than 60 years at transplantation than for younger patients $(p<0.05)$. The 6-year actuarial survival after transplantation for patients older than 60 years was $54 \%$ compared with $72 \%$ for patients younger than 60 years at transplantation $(p<0.05)$. Patients older than 60 years at transplantation were more likely to die of infectious complications or malignant disease after transplantation $(p<0.05)$. We believe caution is warranted in offering cardiac transplantation to patients older than 60 years. This group of patients should be carefully observed for the development of potentially life-threatening infectious complications or new malignant tumors after transplantation. (J THOraC CARdiovasc Surg 1996;111:423-8)

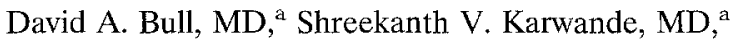
John A. Hawkins, MD, ${ }^{a}$ Leigh A. Neumayer, MD, ${ }^{a}$ David O. Taylor, MD, ${ }^{b}$ Kent W. Jones, MD, ${ }^{\mathrm{a}}$ Dale G. Renlund, MD, ${ }^{\mathrm{b}}$ Charles W. Putnam, MD, ${ }^{\mathrm{c}}$ and the UTAH Cardiac Transplant Program, Salt Lake City, Utah, and Tucson, Ariz.
In the 1960 s and 1970 s, cardiac transplantation was usually restricted to patients younger than 50 years of age. ${ }^{1}$ In the 1980 s, particularly after the introduction of cyclosporine, the likelihood of survival after cardiac transplantation progressively increased. As survival improved, criteria for candidacy became less restrictive. The amended criteria included a progressive relaxation of the upper age limit for

From The Departments of Cardiothoracic Surgery and Cardiology, ${ }^{\mathrm{b}}$ University of Utah School of Medicine, Salt Lake City, Utah, and The Department of Surgery, ${ }^{c}$ University of Arizona School of Medicine, Tucson, Ariz.

Read at the Twenty-first Annual Meeting of The Western Thoracic Surgical Association, Coeur d'Alene, Idaho, June 21-24, 1995.

Address for reprints: David A. Bull, MD, University of Utah Medical Center, 50 North Medical Dr., Salt Lake City, UT 84132.

Copyright (C) 1996 by Mosby-Year Book, Inc.

$0022-5223 / 96 \$ 5.00+0 \quad \mathbf{1 2 / 6 / 6 9 6 6 1}$ recipients, ultimately including patients older than 60 years in many cardiac transplant centers. Support for relaxation of the upper age limit came from several cardiac transplant programs, which reported equivalent 1-year survivals for patients older than 60 years of age. ${ }^{2-6}$ In the Utah Transplant Affiliated Hospitals (UTAH) Cardiac Transplant Program, we have offered cardiac transplantation to patients older than 60 years with end-stage heart failure if they were otherwise acceptable candidates for transplantation. However, because the availability of donor organs for cardiac transplantation is limited, a long-term assessment of the results of cardiac transplantation in patients older than 60 years should be made, examining whether the durable function from each donor heart is being realized when the transplant recipient is older than 60 years. This report examines the long-term results of cardiac transplantation in patients older than 60 years. 


\section{Methods}

The criteria for eligibility as a cardiac transplant recipient in the UTAH Cardiac Transplant Program include (1) severe class IV cardiac dysfunction unremedial to surgical treatment other than cardiac replacement, (2) limited life expectancy, with 1-year mortality estimated to be greater than $50 \%$, (3) physiologic age less than 65 years, with an absolute upper limit for chronologic age of 65 years, (4) no systemic illness other than abnormalities related to heart failure, (5) emotional stability, and (6) strong family support system. Exclusion criteria include (1) severe pulmonary hypertension (pulmonary vascular resistance greater than 6 Wood units with inability of nitroprusside infusion to reduce resistance to less than 4 Wood units or inability to reduce pulmonary artery systolic pressure below $60 \mathrm{~mm} \mathrm{Hg}$ ), (2) severe irreversible hepatic, renal or pulmonary disease, (3) active systemic or pulmonary infection, (4) recent pulmonary infarction, (5) history of uncontrollable hypertension, defined as blood pressure consistently greater than $160 / 100 \mathrm{~mm} \mathrm{Hg}$ with medical therapy, (6) uncorrectable peripheral vascular disease, (7) active peptic ulcer disease, and (8) history of substance abuse (including alcohol) or behavior problem that would interfere with medical compliance.

Carotid duplex studies, pulmonary function tests, chest $\mathrm{x}$-ray films, arterial blood gases, resting multiple gated acquisition scan, echocardiogram, cardiac catheterization, purified protein derivative skin test, and dental consults are obtained on all potential recipients. Colonoscopy is performed on all potential recipients older than 50 years.

Orthotopic cardiac transplants were performed in all patients. No effort was made to match older or less than ideal donor hearts to older recipients. All recipient patients received antibiotic prophylaxis with cefuroxime given at least $1 / 2$ hour before the operation. Antibiotics were added to the pump prime and were administered for 48 hours after the operation.

Early rejection prophylaxis was based on murine monoclonal CD3 antibody (OKT3). Our immunosuppressive protocol involves a 14-day course of OKT3 beginning with a dose during the operation at the time of the pulmonary artery anastomosis. OKT3 levels and CD3 lymphocyte counts are monitored daily for 14 days. Cyclosporine administration is begun on the third or fourth postoperative day at an oral dosage of $6 \mathrm{mg} / \mathrm{kg}$ per day and is adjusted to a target trough level of 150 to $200 \mathrm{ng} / \mathrm{ml}$. Azathioprine (2 to $4 \mathrm{mg} / \mathrm{kg}$ ) is administered before the operation, and the postoperative dosage is adjusted to maintain the white blood cell count between 4 and $6 \times$ $10^{9} / \mathrm{L}$ (4000 and 6000 leukocytes/ml). Methylprednisolone is administered during the operation ( $500 \mathrm{mg}$ intravenously). Corticosteroids (prednisone or equivalent) are then administered at a dose of $0.25 \mathrm{mg} / \mathrm{kg}$ per day until the completion of the OKT3 course, when the dosage is increased to $1 \mathrm{mg} / \mathrm{kg}$ per day for 7 days.

Average doses of cyclosporine and azathioprine were determined for each patient and expressed as milligrams per square meter of body surface area per day. Steroid dosing was calculated as prednisone equivalents, that is, 1 . $\mathrm{mg}$ of prednisone $=0.8 \mathrm{mg}$ of methylprednisolone $=4 \mathrm{mg}$ of hydrocortisone, and included steroids administered to the patient at the time of the operation. Cumulative steroid dosing, expressed as milligrams per kilogram, was determined at each posttransplantation year.

Patients routinely underwent endomyocardial biopsy weekly for 6 to 8 weeks after transplantation. The frequency of biopsy was then decreased in accordance with the status of cardiac rejection. Rejection was treated with high-dose methylprednisolone or prednisone, with or without antithymocyte globulin, OKT3, or rabbit antilymphoblast globulin, followed by gradually decreasing doses of prednisone. After the prophylactic use of prednisone, or after its use in the treatment of rejection, prednisone was gradually decreased and discontinued unless cardiac rejection recurred. The process of prednisone dosage reduction and discontinuation was repeatedly attempted until three rejection episodes within a 4-month period had occurred in a given recipient, at which time the prednisone dosage was maintained at the lowest level necessary to prevent recurrent rejection ( 5 to $20 \mathrm{mg}$ per day).

Rejection was diagnosed from histologic studies of the endomyocardial biopsy specimens. Biopsy specimens were graded by the consensus of three cardiac pathologists. Rejection severity was grade 0 to 4 according to the International Society for Heart and Lung Transplantation grading system. ${ }^{7}$ Grade 2 was managed with an increase in oral steroids. Intravenous bolus steroid therapy was given for grade $3 \mathrm{~A}$ rejection. Patients with grade $3 \mathrm{~B}$ rejection also received cytolytic therapy as indicated. Grade 4 rejection was managed with both cytolytic therapy and intravenous steroids.

The number of serious infections, defined as infections necessitating intravenous antibiotics, was determined for each patient. The infections were categorized according to originating site in all instances. Evidence of prior cytomegalovirus infection in either donor or recipient was treated with prophylactic gancyclovir.

Data regarding age at transplantation, number of male and female patients, indication for transplantation, number of patients whose condition is designated status 1 , length of time on the waiting list, donor heart ischemic time, postoperative immunosuppressive regimens and dosages, number and severity of rejection episodes, number and site of episodes of infection, cause of death, and survival are continuously updated for each transplant recipient in the UTAH Cardiac Transplant Program as part of our ongoing patient database. This information from the database forms the basis for this report.

Survival curves for patients younger than 60 years and older than 60 years at transplantation were established, with mortality as the main outcome variable, by means of the Kaplan-Meier method and compared by the Wilcoxon and log-rank tests. The $\chi^{2}$ test was used to compare qualitative demographic and cause of death data. The $t$ test was used to compare quantitative data between the two groups, reported as the mean \pm standard deviation.

\section{Results}

From 1985 to 1994, 527 patients underwent cardiac transplantation, of whom 101 were older than 60 years of age at transplantation. The mean follow-up of this group of patients is 6 years, with the longest follow-up being 9 years. 
Table I. Comparison of recipient characteristics

\begin{tabular}{lcccccc}
\hline & \multicolumn{2}{c}{ Age $>60 y r$} & \multicolumn{2}{c}{ Age $<60 y r$} & \\
\cline { 2 - 4 } & No. & $\%$ & No. & $\%$ & Value \\
\hline Male & 92 & 91 & 357 & 84 & NS \\
Ischemic cardiomyopathy & 63 & 62 & 213 & 50 & NS \\
Status 1 & 44 & 44 & 179 & 42 & NS \\
\hline
\end{tabular}

NS, Not significant.

Table II. Comparison of recipient characteristics

\begin{tabular}{lccc} 
& & & $p$ \\
& Age $>60 y r$ & Age $<60 y r$ & Value \\
\hline Time on waiting list (days) & $80 \pm 117$ & $74 \pm 82$ & NS \\
$\begin{array}{l}\text { Donor heart ischemic } \\
\text { time (min) }\end{array}$ & $157 \pm 62$ & $170 \pm 60$ & NS \\
$\begin{array}{l}\text { Cumulative steroids at } \\
1 \mathrm{yr}(\mathrm{mg} / \mathrm{kg})\end{array}$ & $12,616 \pm 32,049$ & $9694 \pm 5468$ & $\mathrm{NS}$ \\
\end{tabular}

NS, Not significant

Patients older than 60 years had significantly fewer rejection episodes per patient than those who were younger than 60 years at transplantation $(1.9 \pm$ 1.3 vs $2.6 \pm 1.8, p=0.009$ ). However, no significant difference was noted between the two groups as to the number of male versus female patients, number of patients in status 1 condition, or the incidence of ischemic, idiopathic, or viral cardiomyopathy as the indication for transplantation (Table I). The two groups also did not differ with regard to length of time on the waiting list before transplantation, donor heart ischemic time, or peak or cumulative doses of cyclosporine, azathioprine (Imuran), steroids, or OKT3 (Table II). No difference was detected in the number of infectious complications per patient between the two groups. Older and younger patients did not significantly differ in their ability to be weaned from steroids after transplantation. The prevalence of infectious complications did not vary with the steroid dosage after the operation.

Both the short-term and long-term survivals after transplantation were significantly poorer for patients who were older than the age of 60 years at transplantation than for younger patients $(p<0.05)$ (Fig. 1). Analysis of the actuarial survival curves for the two groups demonstrates divergence of the curves beginning 6 months after transplantation. The difference between the curves becomes more pronounced at 1 year with progressive divergence of the curves thereafter. The 6-year actuarial survival after transplantation for patients older than 60 years was $54 \%$ compared with $72 \%$ for patients operated

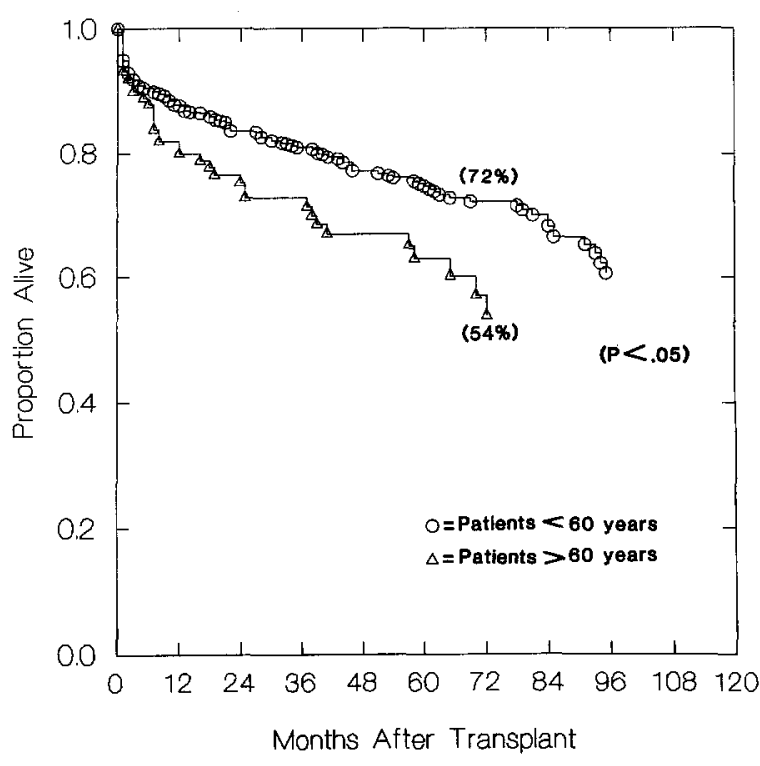

Fig. 1. Actuarial survival curves after transplantation for patients younger than 60 years and older than 60 years at cardiac transplantation.

on when younger than 60 years $(p<0.05)$. Although no statistically significant difference between the two groups was detected with regard to the number of infectious complications per patient, patients older than 60 years were more likely to die of an infectious complication after transplantation $(p \leq 0.003)$. These fatal infectious complications included gramnegative sepsis $(n=7)$, Aspergillus $(n=6)$, Candida $(n=2)$, Nocardia $(n=1)$, mucormycosis $(n=1)$, and hepatitis $\mathrm{C}(n=1)$. There was no difference in the specific causes of fatal infectious complications when comparing the two age groups.

Patients older than 60 years were also more likely to die of malignant disease after transplantation $(p=0.015)$. Among these patients, the fatal malignancies were lymphoma $(n=3)$, leukemia $(n=1)$, carcinoma of the lung $(n=2)$, and carcinoma of the esophagus $(n=1)$. The overall prevalence of posttransplantation lymphoproliferative disorders in our program is $2 \%$. No difference in the specific types of malignant disease surfaced when the two age groups were compared. Patients with a posttransplantation lymphoproliferative disorder were initially treated with a reduction in immunosuppression. If this therapy was unsuccessful, they began chemotherapy.

The two groups did not otherwise differ with regard to the prevalence of acute or chronic rejection or accelerated coronary atherosclerosis as the 
Table III. Comparison of causes of death between groups

\begin{tabular}{lrrrrrr}
\hline & \multicolumn{2}{c}{ Age $>60 y r$} & & \multicolumn{2}{c}{ Age $<60 y r$} & \\
\cline { 2 - 3 } Cause of death & No. & $\%$ & & No. & $\%$ & p Value \\
\hline Infection & 18 & 18 & & 34 & 7 & $\leq 0.003$ \\
Malignancy & 7 & 7 & 8 & 2 & 0.015 \\
Chronic rejection & 8 & 8 & 30 & 7 & NS \\
Acute rejection & 2 & 2 & 12 & 3 & NS \\
Gastrointestinal & & & 12 & 3 & NS \\
Operative & 1 & 1 & 9 & 2 & NS \\
Renal & & & 4 & 1 & NS \\
Pulmonary & & & 3 & 1 & NS \\
Other & 1 & 1 & 6 & 1 & NS \\
\hline
\end{tabular}

cause of death. No significant difference was noted between the two groups as to the prevalence of gastrointestinal, renal, pulmonary, neurologic, or other complications as the cause of death (Table III).

\section{Discussion}

As survival after cardiac transplantation improved, criteria, including age, gradually were relaxed. Cited in support of such policy changes were the results from several centers indicating that survivals were not significantly worse in patients older than 60 years. ${ }^{2-6}$ These reports, however, were limited by small numbers of patients and short-term follow-ups. Long-term survival and functional results become more relevant when the best use of a limited supply of cardiac donors is considered.

Our data show a significantly reduced short-term and long-term survival in transplant recipients older than 60 years. The cardiac recipient older than 60 years has, in our experience, an increased likelihood of death caused by infection and malignancy.

Our observations suggest that physiologic changes take place beyond 60 years of age, which may significantly affect long-term survivals after cardiac transplantation. Older patients have decreased immune responsiveness and $\mathrm{T}$-cell function and, as a group, may require less immunosuppression. ${ }^{5,6}$ Our data and those of others confirm that patients older than 60 years have fewer rejection episodes per patient than recipients younger than 60 years. ${ }^{2,3,6}$ On the other hand, patients older than 60 years are more likely to die of an infectious complication or malignant disease after transplantation. Perhaps a decline in immune responsiveness resulting in fewer rejection episodes in patients older than 60 years may also make them more susceptible to fatal infectious complications or malignant disease after transplantation. An alternative, but not mutually exclusive, hypothesis is that this age group may be susceptible to relative over-immunosuppression. It seems unlikely that differences in mortality between the two groups can be ascribed to latent pathophysiology in the older patients before transplantation, because older patients were subjected to the same, or even more rigorous, selection criteria as the younger patients. Moreover, we did not observe any increase in death from other causes typically seen in older patients, such as renal, cerebrovascular, pulmonary, or gastrointestinal disease (Table III).

This information is important when future guidelines are considered for selection of patients older than 60 years for cardiac transplantation. Improvements in immunosuppression and other aspects of management have achieved acceptable short-term survivals in a variety of potential recipients at high risk, including patients older than 60 years. Relaxation of criteria or candidacy and hence expansion of the recipient pool, however, stresses an already inadequate donor supply. Careful review of the likely long-term outcome of a potential recipient should be undertaken to ensure that donor hearts will most effectively benefit the entire group of potential transplant recipients. At present, we remain highly selective in accepting patients older than 60 years as potential cardiac transplant recipients. The presence of any of the exclusion criteria discussed herein contraindicates transplantation. Once these patients are accepted, however, we do not penalize patients older than 60 years or assign them a lower priority score on the basis of their age, nor do we attempt to match older donor hearts to older recipients.

Analysis of our long-term actuarial survival after cardiac transplantation leads us to speculate that cardiac transplantation may be of limited utility for the management of end-stage heart failure in patients older than 60 years, unless improvements in, or tailoring of, immunosuppressive regimens reduce the prevalence of late infectious and malignant complications. If this can be accomplished, while maintaining an acceptable risk of rejection, then improvement in long-term survival may be possible in the patient older than 60 years.

Nonetheless, we maintain that cardiac transplantation, when offered to patients older than 60 years, must be prefaced by strict criteria for candidacy and followed by rigorous surveillance to detect and treat potentially fatal infectious complications and malignant diseases. 


\section{REFERENCES}

1. Copeland JG, Stinson EB. Human heart transplantation. Curr Probl Cardiol 1979;4:1-51.

2. Frazier OH, Macris MP, Duncan JM, Van Buren CT, Cooley DA. Cardiac transplantation in patients over 60 years of age. Ann Thorac Surg 1988;45:129-32.

3. Miller LW, Vitale-Noedel N, Pennington G, McBride L, Kanter KR. Heart transplantation in patients over age fifty-five years. J Heart Transplant 1988;7:254-7.

4. Fabbri A, Sharples LD, Mullins P, Caine N, Large S, Wallwork J. Heart transplantation in patients over 65 years of age with triple-drug therapy immunosuppression. J Heart Lung Transplant 1992;11:929-32.

5. Heroux AL, Costanzo-Nordin MR, O'Sullivan JE, et al. Heart transplantation as a treatment option for end-stage heart disease in patients older than 65 years of age. J Heart Lung Transplant 1993;12:573-7.

6. Renlund DG, Gilbert EM, O'Connell JB, et al. Ageassociated decline in cardiac allograft rejection. Am J Med 1987;83:391-8.

7. Billingham ME, Cary NR, Hammond ME, et al. A working formulation for the standardization of nomenclature in the diagnosis of heart and lung rejection: Heart Rejection Study Group. J Heart Transplant 1990;9:587-92.

\section{Discussion}

Dr. Robert C. Robbins (Stanford, Calif.). Dr. Bull's data are important to consider in this economically efficient environment. Several centers, including the Utah group, have previously reported that the 1-year survival for older cardiac transplant recipients was equivalent to survival in younger patients. This is the first report to describe the intermediate-term results for older cardiac recipients. The 6 -year actuarial survival for patients older than 60 years is clearly lower than for patients younger than 60 years, with the actuarial curves beginning to diverge 1 year after transplantation. The actuarial survival of 35 cardiac transplant recipients older than 60 years operated on at Stanford over the past 10 years was the same as the survival of younger patients during this same period. Of the 10 deaths in these older patients, two were the result of infection and one the result of a nonlymphoid malignancy. One would expect that actuarial survival decreases with age, but this paper focuses on malignancy and infection as the primary causes of death.

Serious infection is defined as an episode requiring intravenous antibiotics. There is no information detailing the types of infections observed. Specifically, I would be interested in the incidence of bacterial, viral, and fungal infections. Was cytomegalovirus prophylaxis used as a routine and, if so, for which subsets of patients?

The incidence of malignancy increases with age and the cancer-related deaths observed in the older patients may be explained on this basis. Because induction therapy with OKT3 was used, it would be interesting to see a more detailed description of the types of cancer that developed in these patients. What was the prevalence of lymphoproliferative disorders and how were these managed? Was colonoscopy performed as part of the preoperative evaluation in the older patients?

The older patients had fewer rejection episodes than the younger recipients. It would be helpful to see a stratification of the grades of rejection for each group, which grades of rejection were treated, and the correlation of treatment with the development of infection and malignancy.

Because the older patients have less rejection, are we all over immunosuppressing this population? What percentage of patients in each group stopped receiving steroids? Would it be reasonable to eliminate induction therapy in this group of less immunocompetent patients?

The concept that older patients may be better served with the implantation of a left ventricular assist device is attractive; however, this approach should be used with caution because the survival of older patients receiving left ventricular assist device support is less than that of younger patients. The recent effort by John Wallwork and associates in Cambridge to use the Novacor left ventricular assist system as an alternative to transplantation for older patients will be extremely useful in providing data concerning this novel treatment.

Dr. Bull. We have been aggressive about performing transplantation in recipients older than 60 years. Nearly $20 \%$ of our transplant population has been older than 60 years. This compares with $11 \%$ in the Stanford experience. Inasmuch as older patients were subjected to the same or more rigorous candidacy criteria as younger patients, I do not believe that our patient selection is the explanation for the less favorable long-term survival in older patients. This is evidenced by the lack of a significant difference in the incidence of renal, pulmonary, gastrointestinal, and neurologic complications between the two groups.

In 1987, we published data regarding the age-associated decline in cardiac allograft rejection. This paper had a small number of patients with short-term follow-up. The conclusion of the paper was that survival was equal between older and younger patients at 1-year follow-up. We did not start to see a difference in survival until we had a larger number of patients whom we had followed up for a longer period after transplantation.

With regard to infection, a number of recipients older than 60 years died 6 to 12 months after transplantation of fungal infections, particularly Aspergillus. After 1 to 2 years, a number of older recipients died of gram-negative sepsis. We are unable, however, to make statistical conclusions regarding the specific cause of infection and mortality. The only conclusion we are able to draw at this time is that mortality from infection in patients older than 60 years is greater than that in younger patients. Our purpose with this study was not to delineate the specific cause of infection or malignancy but rather to compare the overall survival of our transplant population on the basis of age. We believe such an analysis is helpful to determine the best use of donor hearts, which are in limited supply for both older and younger recipients.

Colonoscopy is part of our routine screening program in potential recipients older than 50 years. Lymphoproliferative disorders were the leading cause of malignancy in our recipients older than 60 years. Our first- 
line of treatment for patients with a posttransplantation lymphoproliferative disorder is to reduce their immunosuppression. If this is unsuccessful, the patients begin chemotherapy. After lymphoproliferative disorders, the most common types of malignancy were solid organ tumors.

We use the International Society for Heart and Lung Transplantation grading system for rejection. Patients with grade 2 rejection are treated with an increase in oral steroids. Patients with grade $3 \mathrm{~A}$ rejection are treated with intravenous bolus steroid therapy. Patients with grade $3 \mathrm{~B}$ rejection may also receive cytolytic therapy. Patients with grade 4 rejection are treated with both cytolytic therapy and intravenous steroids.
It was not possible from our analysis to assess whether the older patients were excessively immunosuppressed. We have looked at subsets of T cells in older patients. As you know, T effector cell-mediated immunity is the primary arm of the immune system involved in allograft rejection. We have not been able to determine any numeric differences in subpopulations of $\mathrm{T}$ cells between older and younger patients. The increased mortality from infection and malignancy, however, may be due to immunosenescence. This term describes the decreased mitogenic response to an antigenic stimulus with aging, resulting in a functional decrease in T-cell activity. We still have much to learn about the changes in the immune system with aging.

\section{Availability of JourNaL back issues}

As a service to our subscribers, copies of back issues of THE Journal of THORACIC AND CARDiovascular SURGERY for the preceding 5 years are maintained and are available for purchase from the publisher, Mosby-Year Book, Inc., at a cost of $\$ 13.50$ per issue. The following quantity discounts are available: $25 \%$ off on quantities of 1.2 to 23 , and one third off on quantities of 24 or more. Please write to Mosby-Year Book, Inc., Subscription Services, 11830 Westline Industrial Drive, St. Louis MO 63146-3318, or call $800-453-4351$ or 314-453-4351 for information on availability of particular issues. If unavailable from the publisher, photocopies of complete issues are available from University Microfilms International, 300 N. Zeeb Rd., Ann Arbor, MI 48106, 313-761-4700. 